\title{
Low-grade fibromyxoid sarcoma: a rare condition with high proliferation
}

\author{
Marzieh Karimi Khezri ${ }^{1}$, M. Özgür Taşkapılığlư ${ }^{1}$, Alper Türkkan ${ }^{2}$, Ulviye Yalçınkaya ${ }^{3}$, Ahmet \\ Bekar $^{1}$
}

${ }^{1}$ Department of Neurosurgery, Uludağ University School of Medicine, Bursa, Turkey

${ }^{2}$ Department of Neurosurgery, VM Medical Park Hospital, Bursa, Turkey

${ }^{3}$ Department of Surgical Pathology, Uludağ University School of Medicine, Bursa, Turkey

\begin{abstract}
Low-grade fibromyxoid sarcoma (LGFMS) is a type of high metastatic potential of the fibrosarcomas. Most of the time there is a long interval between tumor presentation and metastasis. We present 2 cases of LGFMS. The first is a 31-year-old female with a mass in anterior aspect of her left thigh, and the other is a 68 -year-old female with mass in posterior of her neck. Both cases underwent operation for several times and confirmed as LGFMS histopathologically, there is no exact protocol for postoperative follow-up to detect early metastases according to the relative variety of LGFMS. So informing the patients about the long-standing metastatic potential of their disease is important.
\end{abstract}

Eur Res J 2018;4(3):238-241

Keywords: fibrosarcoma, low-grade fibromyxoid sarcoma, surgery

\section{Introduction}

No Low-grade fibromyxoid sarcoma (LGFMS) is a differentiated sub-type of fibrosarcoma. Evans first described this pathologic entity as a rare high metastatic potential soft-tissue tumor despite its benign histologic appearance in 1987 [1]. Usually pathologists, radiologists, and surgeons fall in problem for fundamental principle for tumor management due to long interval period between tumor presentation and metastasis behavior. It is still difficult to estimate LGFMS incidence because many cases are not diagnosed as LGFMS. Although LGFMS can be found in unusual places, like head, retroperitoneum,or the chest wall, these tumors usually occur in the proximal extremities and trunk [2,3]. Subfascial location is the most common place for LGFMS occurrence, as subcutis or dermis may be affected in rare occasions [4]. LGFMS especially happen in young to middle-age adults, by the way a large member of cases have been reported in pediatric ages $[3,5,6]$. In this report, we presented 2 cases of LGFMS; a 31-year-old female with a mass in anterior aspect of her left thigh, and a 68-year-old female with a mass in posterior part of her neck. 


\section{Case Presentation}

\section{Case 1}

A 31-year-old female presented with a recurrent large tumor mass on the anterior aspect of her left thigh operated for three times before admitted to our clinic. She had been operated due to a similar tumor presentation 16 and 14 years ago at another medical center, and 6 years ago at our clinic. The mass has been growing slowly during past 6 years, but the patient did not seek for medical treatment until the tumor size disturbed her regular life. Physical examination revealed firm, multifocal, mobile masses without tenderness, redness, or warmth (Figure 1a). Laboratory evaluations and plain radiographs were unremarkable. Magnetic resonance imaging (MRI) demonstrated a contrast enhanced tumor with myxoid and fibrous pattern (Figures $1 \mathrm{~b}$ and $1 \mathrm{c}$ ). The tumoral masses were excised. Due to infiltration of muscular and fatty tissues, quadriceps femoris and adductor longus muscles were excised (Figures $1 \mathrm{~d}$ and 1e). Medial and lateral parts of femoral bone periosteum were invaded by tumor and resected. After surgery the patient experienced no major complication and was discharged 5 days after the operation. The histopathological diagnosis was LGFMS (Figure 1f).

\section{Case 2}

A 68-year-old female patient presented with large recurrent tumoral masses on posterior part of her neck, radiating to her shoulders, and upward of right shoulder. During past 25 years she has been operated 3 times because of neck masses. Physical examination revealed neck stiffness and firm multifocal mobile masses especially radiated to right shoulder. There was no impairment of sensory and motor nerves. Cervical MRI demonstrated multiple sized and firm contrast enhanced masses which spread to both shoulders (Figure 2). The detected masses were excised. Periosteal infiltration was observed at spinous

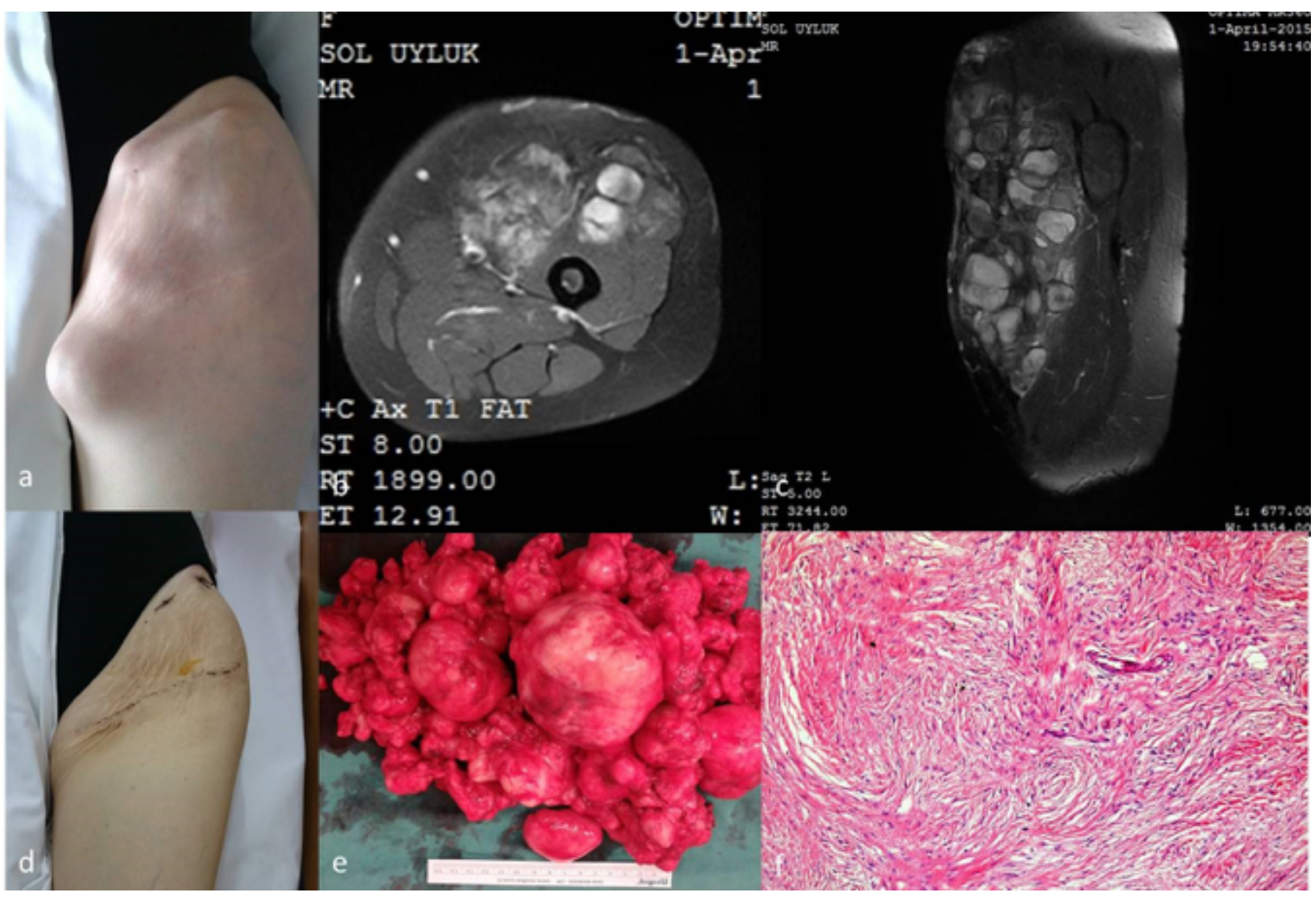

Figure 1. (a) Preoperative left leg mass; (b, c) The fibrous components were identified as hypointense area on T1- and T2-weighted MR images and slightly enhancing on T1-weighted MR images after intravenous administration. The myxoid components of the mass were recognized as hypointense on T1-weighted MR images and hyperintense on T2-weighted MR images, and enhancing on T1-weighted MR images after contrast administration; (d) Postoperative appearance of the patient's leg; (e) The excised specimen. The surface of the tumoral mass was smooth and glistering with white-gray color. There was no lenfovascular invasion or necrotic area; (f) Histopathological appearance of the mass. Immunohistochemistry study of the tumor revolves; EMA: focal positive, SMA: focal positive, MSA: negative. P53: negative, Ki 67: 3\% positive. 


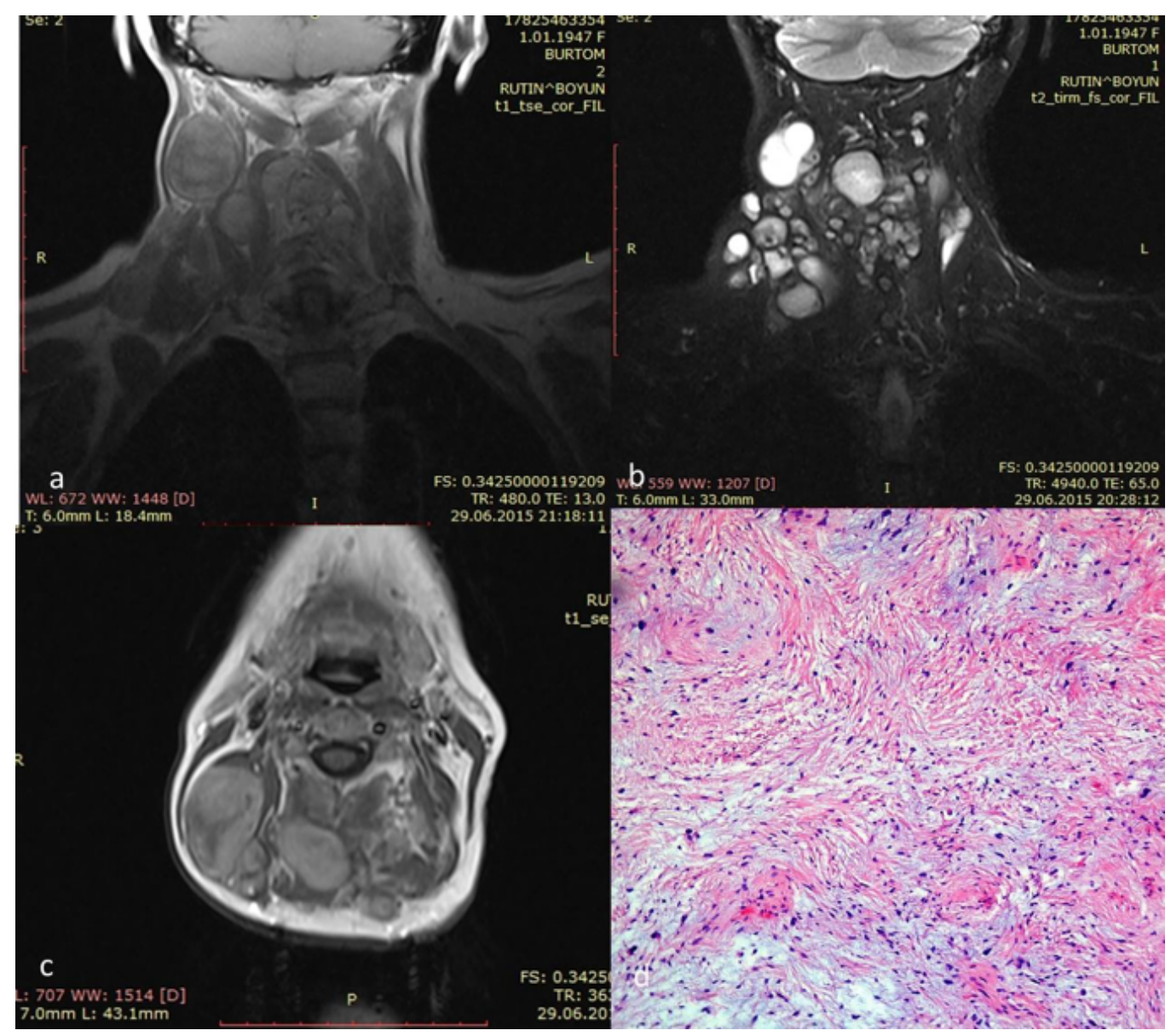

Figure 2. (a-c) C6 and C7 laminar and spinal process involvement was seen at MRI. Enhancing after contrast administration was detected as in the first case; (d) Nodular shape infiltration of hyperchromatic, pleomorphic nucleus of mesenchymal cells surrounded with fibrous stroma reported in pathological sections. Also diffused amyloid degeneration reported in tumoral section. Study of the tumor revolve; S100: strongly positive, Vimentin: strongly positive, SMA: focally positive, Desmin: strongly positive.

processes of $\mathrm{C} 6$ and $\mathrm{C} 7$ and vertebral lamina was resected together with infiltrated parts of bone. After surgery, the patient experienced no major complication and discharged 2 days after surgery. The histopathological evaluation was reported as LGFMS (Figure 2).

\section{Discussion}

TLGFMS manifestation is usually long-standing and depended on the anatomic area of the lesion. Mostly its presentation is as painless soft tissue masses with duration of over 5 years in 5\% of patients [2]. Acute presentation of LGFMS is rare, and it can happen in chest wall infiltration as acute respiratory distress syndrome (ARDS) or chest pain. It may be presented with seizure when intracranial infiltration is suspected $[3,7]$. In this paper we presented two cases, diagnosed at 16 years and 25 years ago.

Special MRI and CT finding for LGFMS are defined, although imaging findings are nonspecific [3,
8-10]. On CT images without contrast, the fibrous structure of these tumors interprete data density of muscular tissue, and the myxoid part was evaluated as hypodense. The fibrous structure was interpreted as hypointense on T1- and T2-weighted images, and there was a slight contrast enhancement on T1weighted MRI images. On the other hand, the myxoid part has been found as hypointense on T1- and hyperintense on T2-weighted images, and strongly contrast enhancing on T1-weighted images. Sometimes calcification can be detected [10]. Both of our cases had contrast enhancement at radiological examination similar to literature.

LGFMS is a sub-type of fibrosarcoma, characterized by a mixture of hypercellular myxoid nodules in collagenized area with low cellularity [6]. Tumoral parts are commonly characterized by round to ovoid nuclei small cells without nucleoid and mildly eosinophilic cytoplasm. Mitosis increased at atypical region determined by hypercellularity; only in $10 \%$ of the cases nuclear hyperchromatism, and necrosis has been reported. Tumor cells are commonly determined 
by absent of sparse mitotic finding, nuclear anaplasia or necrosis. It is positive for vimentin and negative for other antibodies, such as S100 protein, desmin, ceratin, epithelial membrane antigen such as CD31, and CD34 at most cases. Lesions showing proliferation of spindle cell with or without fibrous component in myxoid pattern are evaluated at differential diagnosis of LGFMS [11]. Tumors with both fibrous and myxoid pattern include fibromatosis, neurofibroma, malignant peripheral sheath tumor, perineurioma, fibrous histiocytoma, or the tumors with only predominant myxoid areas without fibrosis such as myxomas, myxoid neurofibroma, angiomyxoms, myxoid liopsarcoma, and low-grade myxofibrosarcoma have to be evaluated at differential diagnosis [10]. Also, desmoid tumors such as desmoplastic fibrosarcoma, and low-grade differentiated liposarcoma should be remembered in the differential diagnosis of LGFMS. If tumor has been removed completely, it is not difficult to diagnose LGFMS due to morphologic pattern and immune phenotypic features. In such cases, an excisional biopsy should be performed before surgical resection according to the fact that it is not commonly possible to diagnose with needle core biopsy or fine needle aspiration. If the diagnosis still remains unclear for myxoid, this kind of cytogenetic for rare cases of LGFMS can be beneficial [4].

Goodlad et al. [5] pointed that LGFMS were inconsistently aggressive tumor. Although all the cases were primarily diagnosed and treated as benign lesions in retrospective study, local recurrence was reported as $68 \%$, and the death rate was reported $18 \%$ [5]. It is clear that patient selection has affected the rate of metastases and recurrence rate because most of them selected according to unexplained metastases. In another large series death rate, local recurrence, and metastasis was detected as $2 \%, 54 \%$, and $6 \%$; respectively [6]. There was no significant relation between recurrence or metastasis and the presentation of high cellularity focal area, nuclear enlargement, increased mitotic activity, and necrosis. Because of potential of late metastasis of LGFMS, as reported 45 years after primer diagnosis, these patients had to be followed for a long period of time [4]. After initial diagnosis of LGFMS the patients must be follow-up by expert oncological group. However exact interval for periodic chest imaging is unclear, as the most metastatic area is lung, several chest CT scan have to be performed during long-term follow-up of these cases.

\section{Conclusions}

The 2 cases report presented herein, enriches the literature for the best diagnosis and surgical treatment of this rare tumor. In addition, it is important to inform patients about long-time metastatic potential of the disease as there is no dedicated protocol regarding follow-up examinations for early metastatic mass diagnosis.

\section{Informed consent}

Written informed consent was obtained from the patient for the publication of this case report.

\section{Conflict of interest}

The authors declared that there are no potential conflicts of interest with respect to the research, authorship, and/or publication of this article.

\section{References}

[1] Evans HL. Low-grade fibromyxoid sarcoma. A report of two metastasizing neoplasms having a deceptively benign appearance. Am J Clin Pathol 1987;88:615-9.

[2] Folpe A, van den Berg E, Molenaar WM. Low grade fibromyxoid sarcoma. In: Fletcher CDM, Unni KK, Mertens F (eds). World Health Organization Classification of Tumours. Pathology and Genetics of Tumours of Soft Tissue and Bone. IARC Press: Lyon, 2002, pp. 104-5. [3] Steiner MA, Giles HW, Daley WP. Massive low-grade fibromyxoid sarcoma presenting as acute respiratory distress in a 12-year-old girl. Pediatr Radiol 2009;39:396-9.

[4] Wu X, Petrovic V, Torode IP, Chow CW. Low grade fibromyxoid sarcoma: problems in the diagnosis and management of a malignant tumour with bland histological appearance. Pathology 2009;41:155-60. [5] Goodlad JR, Mentzel T, Fletcher CD. Low grade fibromyxoid sarcoma: clinicopathological analysis of eleven new cases in support of a distinct entity. Histopathology 1995;26:229-37.

[6] Folpe AL, Lane KL, Paull G, Weiss SW. Low-grade fibromyxoid sarcoma and hyalinizing spindle cell tumor with giant rosettes: a clinicopathologic study of 73 cases supporting their identity and assessing the impact of high-grade areas. Am J Surg Pathol 2000;24:1353-60.

[7] Saito R, Kumabe T, Watanabe M, Jokura H, Shibuya M, Nakazato $\mathrm{Y}$, et al. Low-grade fibromyxoid sarcoma of intracranial origin. J Neurosurg 2008;108:798-802.

[8] Kim SY, Kim M, Hwang YJ, Han YH, Seo JW, Kim YH, et al. Low grade fibromyxoid sarcoma CT, sonography, and MR findings in 3 cases. J Thorac Imaging 2005;20:294-7.

[9] Fujii S, Kawawa Y, Horiguchi S, Kamata N, Kinoshita T, Ogawa T. Low-grade fibromyxoid sarcoma of the small bowel mesentery: computed tomography and magnetic resonance imaging findings. Radiat Med 2008;26:244-7.

[10] Mohamed M, Fisher C, Thway K. Low-grade fibromyxoid sarcoma: Clinical, morphologic and genetic features. Ann Diagn Pathol 2017;28:60-7.

[11] Rakheja D, Seaward JR, Timmons CF. Low-grade fibromyxoid sarcoma with striking zonation. Int J Surg Pathol 2017:1066896917742724. 